\title{
Photonic Microstructures for Gas Detection in the MIR
}

\author{
C. Kraeh ${ }^{1,2}, \underline{\text { A. Popescu }}{ }^{1}$, J. L. Martinez-Hurtado ${ }^{2}$, J. J. Finley ${ }^{2}$ \\ ${ }^{1}$ Siemens AG, Corporate Technology, Otto-Hahn-Ring 6, D-81739 München \\ popescu.alexandru@siemens.com \\ ${ }^{2}$ Walter Schottky Institut, Technische Universität München, D-85748 Garching
}

\begin{abstract}
This paper reports on the fabrication and the investigation of photonic microstructures, which are capable of enhancing the sensitivity of spectroscopic gas sensors in the mid-infrared spectral region, and thus provides routes towards fully integrated and miniaturized on-chip solutions. The presented photonic crystal structures consist of high aspect ratio microtube arrays. The microtubes are produced by etching an array of macropores into a silicon substrate using a photoassisted electrochemical etching technique, subsequently filling these pores via chemical vapor deposition and removing the silicon substrate via anisotropic etching. These photonic crystal structures are optimized for strong transmittance in the mid-infrared from 5-8 $\mu \mathrm{m}$, whereas a major part of the electromagnetic density propagates in-between the microtubes, where it can be absorbed by gas molecules. By using slow light effects, i.e. reduced group velocities of the propagating light, absorption by gas molecules was enhanced by roughly a factor of 10 inside the photonic crystal.
\end{abstract}

Key words: spectroscopy, photonic crystal, mid-infrared, slow light

\section{Introduction}

Optical gas sensors combine high selectivity of target gases with high accuracies. The mid and near-infrared spectral range of the electromagnetic spectrum is of particular interest, as a large variety of technical important gases absorb light there [1]. In order to profit from the outstanding advantages of this technique on a widely available basis, compact and integrated devices are needed. Generally speaking, gas spectrometers measure the absorption of light by atomic or molecular gases along a predefined path. Their sensitivity strongly depends on the pathlength through the gas volume and the absorption cross-sections of the gases. As the latter are fundamental, conventional gas spectrometers increase their sensitivity by guiding the light beam multiple times through a gas volume via reflections; commonly referred to as multipass cells [1]. Most multipass cells offer typical cell volumes of $0.3-10$ liter and pathlengths $>10 \mathrm{~cm}$ [1]. These sensors, although very sensitive to small gas concentrations, are difficult to miniaturize, since a size reduction often leads to a reduction of the interaction path through the gas volume, and hence also to a reduction in sensitivity. In addition, big multipass cells can suffer from reliability issues due to deviations in the alignment of the mirrors, and optical interferences, such as Fabry-Perot etalons, leading to limitations in the sensor performance [1]. In this work, a miniaturized gas spectrometer that mitigates these issues is presented. The integration of a photonic crystal $(\mathrm{PhC})$ structure into the sensor allows the exploitation of a reduced group velocity of light through the PhC. These slow light effects are used to increase the interaction time of the light with the gas molecules, and thus enhance the absorption signal. This increases the sensitivity of the sensor and allows significant size reductions [2].

\section{Fabrication of high aspect ratio microtube arrays}

The PhCs were realized by a fabrication technique based on (1) electrochemical etching of macroporous silicon (Si), (2) subsequent pore filling via deposition from the gas phase, and (3) selective removal of the Si substrate [3]. Fig. 1a-d shows schematics of the fabrication process. 6 inch n-doped (100) Si wafers were used as substrate material. The substrates were phosphor doped and had resistivities of 5 , 20 , and $93 \Omega \mathrm{cm}$. First, a substrate was covered by a silicon nitride $\left(\mathrm{Si}_{3} \mathrm{~N}_{4}\right)$ layer via low pressure chemical vapor deposition (LPCVD) into which an array of windows was defined via photolithography. By immersion into 33wt $\%$ potassium hydroxide $(\mathrm{KOH})$ at $80^{\circ} \mathrm{C}$ etch pits were produced at the window sites (see Fig. 1a). The $\mathrm{Si}_{3} \mathrm{~N}_{4}$ layer was then removed by a 
Fabrication methods:

a) Etch pits

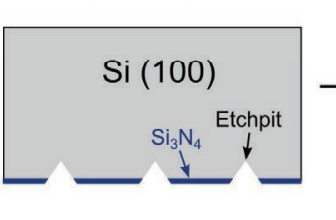

b) Pore etching c) Oxynitride + poly-Si

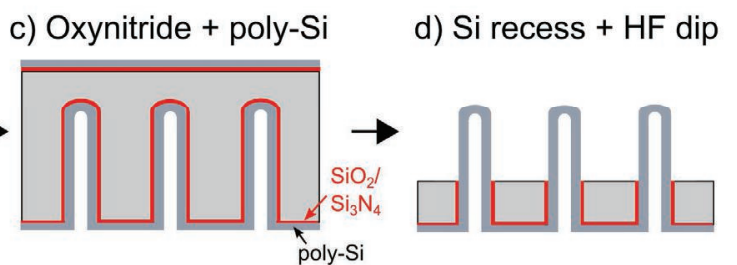

Microtube arrays:

e) Hexagonal array

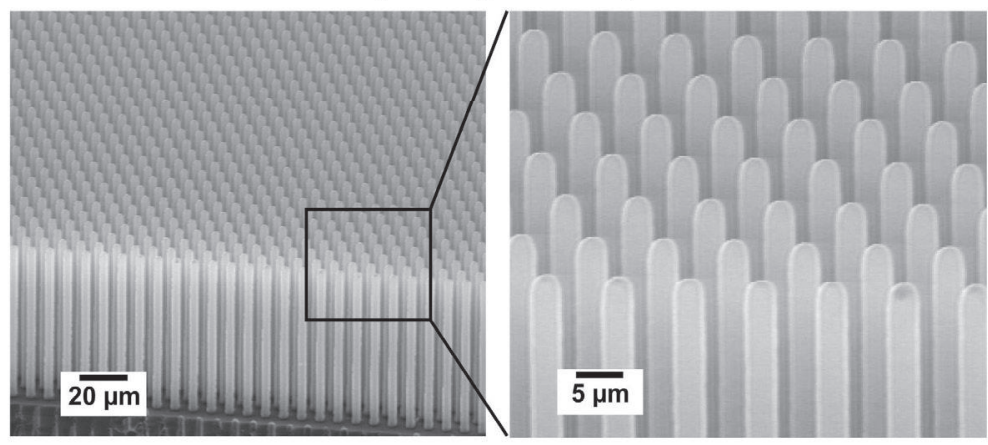

f) Microtube cross-section

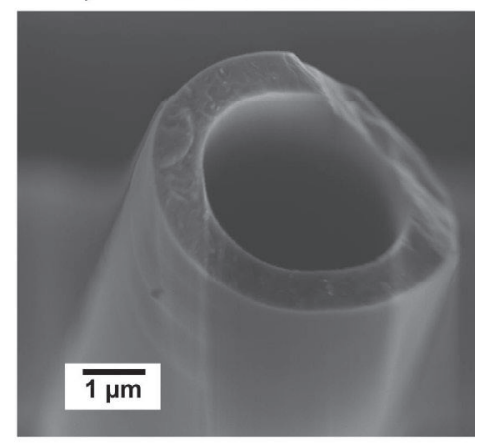

Fig. 1: Fabrication technique and resulting microtube arrays. Top: Schematic of the fabrication process: a) Etchpits are defined into the Si substrate via photolithography and $\mathrm{KOH}$ etching. b) Pores are etched at these sites via a photoassissted etching technique. c) The pores are subsequently filled by a passivation layer and a poly-Si filling. d) The Si substrate is partially recessed and the passivation is removed. Bottom: SEM micrographs of microtube arrays. e) A hexagonal microtube array with a pitch of $6.8 \mu \mathrm{m}$ and a microtube height of $80 \mu \mathrm{m}$. f) Cross-section of a microtube.

short dip in $10 w t \%$ hydrofluoric acid (HF). The etch pits serve as initiation sites for the pores that were produced by a photoassissted electrochemical etching technique (see Fig. 1b) [4]. In this way, an array of high aspect ratio pores was etched into the Si substrate; these pores served as mold for the later microtube array. To increase the pore diameter and reduce their surface roughness a $300 \mathrm{~nm}$ layer of silicon oxide $\left(\mathrm{SiO}_{2}\right)$ was grown inside the pores via thermal oxidation and subsequently removed by a HF (10wt\%) dip. The pores were subsequently filled with a passivation layer consisting of $300 \mathrm{~nm}$ of $\mathrm{SiO}_{2}$ and $80 \mathrm{~nm}$ of $\mathrm{Si}_{3} \mathrm{~N}_{4}$ via thermal oxidation and LPCVD, respectively. On top of this passivation, a 300$350 \mathrm{~nm}$ thick layer of undoped poly-Si was deposited via LPCVD (see Fig. 1c). Subsequently the Si substrate was partially recessed via anisotropic etching with 33wt $\%$ $\mathrm{KOH}$ at $80^{\circ} \mathrm{C}$, exposing an array of microtubes buried in a stabilizing substrate. The microtubes are protected from the $\mathrm{KOH}$ due to their passivation layer which was subsequently removed by an HF (10wt\%) dip (see Fig. 1d). Finally, the substrate was diced into chips of $1.5 \times 10 \mathrm{~mm}^{2}$ size for optical characterization using a Nd:YAG laser; the laser cut predetermined breaking lines into the substrate backside, along which the wafer was carefully separated using tweezers. For optical characterization and gas measurements, square and hexagonal microtube arrays with pitches between 6.1 and $6.8 \mu \mathrm{m}$ and heights of $80 \mu \mathrm{m}$ were fabricated. SEM images of these microtubes are shown in Fig. 1e and 1f. The micrographs reveal a high uniformity and low surface roughness along the microtube length. Microtubes with aspect ratios of up to 1:50 could be fabricated using this technique [3].

A key step in this fabrication process is the etching of the macropores in Fig.1b that define the geometry of the resulting microtubes. The pore etching is based on a photoassissted electrochemical etching process [4] that is schematically depicted in Fig. 2a. A 6wt\% HF solution is used as etchant. The dissolution of Si by HF is supported by defect electrons that are injected into the substrate via backside illumination. The defect electrons are accelerated towards the Si/HF-interface via an applied voltage. The resulting etching current drives the pore growth, which is stabilized by a 


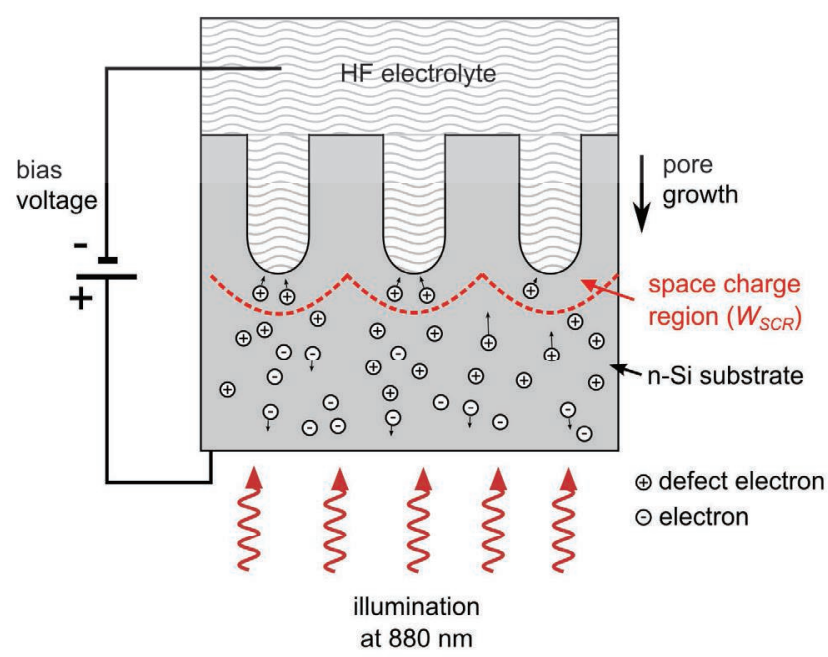

b) Macropore arrays: geometry vs substrate resitivity

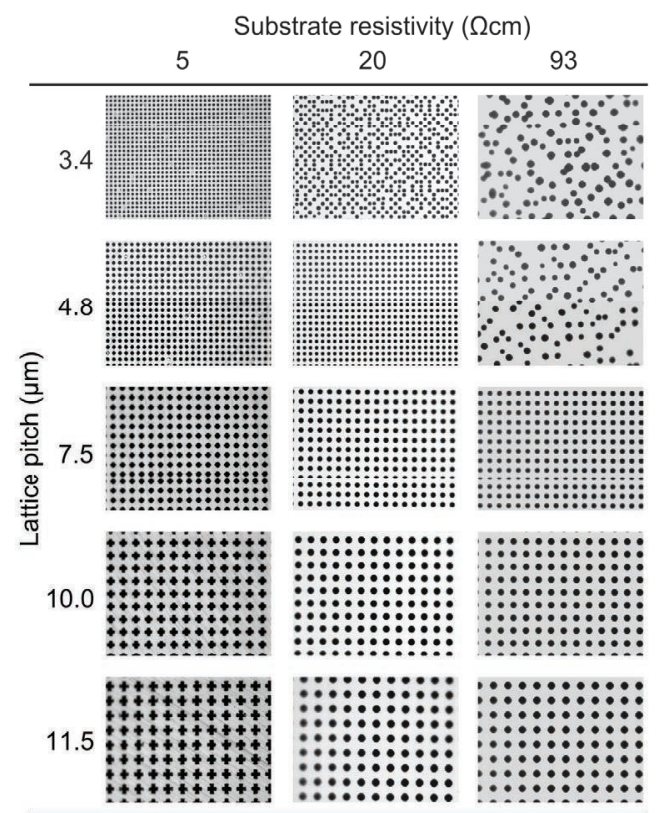

Fig. 2: Photoassissted pore etching. a) Schematic of the etching process. Minority charge carriers (defect electrons) are injected into the Si substrate by backside illumination at $880 \mathrm{~nm}$ and accelerated towards the Si/HF interface by an applied voltage. A space charge region builds around the growing pores, that guarantees that the defect electrons reach the HF at the bottom of the pores. b) Square arrays with pitches between 3-12 $\mu$ m etched in Si substrates with resistivites of 5, 20, and $93 \Omega \mathrm{cm}$, respectively. Stable pore growth at low lattice pitches is not possible for high resistivity.

space charge region of size $W_{\mathrm{SCR}}$ that builds up at the Si/HF-interface. $W_{\mathrm{SCR}}$ depends on the applied bias voltage $V_{\text {appl }}$ and the doping density of the substrate $N_{D}$ :

$$
W_{S C R}=\sqrt{\frac{2 \varepsilon_{S i} \varepsilon_{0} V}{e N_{D}}},
$$

with $V=V_{b i}-V_{a p p l}-k T / e$, where $V_{b i}$ is the built-in potential of the $\mathrm{Si}$ electrolyte interface $(\sim 0.5 \mathrm{~V}), \varepsilon_{0}$ the dielectric constant of vacuum and $\varepsilon_{S i}$ the relative dielectric constant of $\mathrm{Si}$.

This space charge region guarantees that all charge carriers of the etching current reach the growing pores at the tip and no charge carriers exist in-between the pores, leading to a uniform pore growth. There are limitations in the geometry of pore arrays that can be processed in substrates with certain resistivities [4]. A systematic study of the etching process with different pore geometries and substrate resistivities was performed, to identify suitable substrates for different lattice pitches in the range from 3-12 $\mu \mathrm{m}$. Therefore, square lattices with pitches of $3.4,4.8,7.5,10.0$, and $11.5 \mu \mathrm{m}$ were etched into three different $n-S i$ substrates with resitivities of 5,20 , and $93 \Omega \mathrm{cm}$ respectively. After the pore etching the substrates were either grinded or oxidized and recessed in $\mathrm{KOH}$, to reveal the pore cross- sections. Fig. $2 \mathrm{~b}$ shows micrographs of the pore cross-sections of all investigated pitch/substrate combinations. Pore arrays with pitches $\geq 7.5 \mu \mathrm{m}$ can be uniformly etched in all three substrates. A limitation exists for lattice pitches of 3.4 and $4.8 \mu \mathrm{m}$. Only the $5 \Omega \mathrm{cm}$ substrate allows uniform etching of all lattice pitches; the lower limit for the $20 \Omega \mathrm{cm}$ and $93 \Omega \mathrm{cm}$ substrates are $4.8 \mu \mathrm{m}$ and $7.5 \mu \mathrm{m}$ respectively. Defects and missing pores occur below these lattice pitch values. The pore cross-section shape of the most uniform arrays is a square with round edges. However, for bigger pitches and low restivities, e.g. for pitches above $10 \mu \mathrm{m}$ in the $5 \Omega \mathrm{cm}$ substrate, the pore cross-section becomes cross-shaped.

The correlation between pore arrays and substrate resistivity is caused by the space charge region $W_{S C R}$ that builds up during the photoassisted etching, as depicted in Fig. 2a. The lower limit of the lattice pitch is caused by the size $W_{\mathrm{SCR}}$. For high substrate resitvities, and corresponding low $N_{D}$ values, $W_{S C R}$ no longer follows the contour of the growing pores and as a consequence, stable pore growth does not occur for all pores in the array, resulting in either defects in the pore arrays or a completely random distribution of pores and pore cross-sections [5]. However, if $W_{S C R}$ is smaller than half the pore distance, charge carriers exist in-between the pores leading to additional etching from the side orthogonal to 
the growth direction [4]. This effect causes the cross-like shape observed in Fig. $2 b$. For the gas measurements presented in this work, we used a hexagonal array of microtubes with a pitch of $6.5 \mu \mathrm{m}$. This structure could be successfully fabricated in substrates with resistivities of 5 and $20 \Omega \mathrm{cm}$.

\section{Methods for optical characterization and gas sensing}

A transmission setup optimized for measurements in the MIR from 5-8 $\mu \mathrm{m}$ was used to perform optical characterization and gas measurements with the PhC samples. The setup is based on a thermal light source (globar), a grating spectrometer, and a mercury cadmium telluride detector. It allows recording transmission spectra of PhCs. A polarization filter was used to restrict the radiation to TM polarized light, due to the polarization dependence of the $\mathrm{PhC}$ band structure. To prevent absorption of the MIR radiation from the globar by atmospheric gases, especially $\mathrm{H}_{2} \mathrm{O}$, the setup was flushed by gaseous nitrogen $\left(\mathrm{N}_{2}\right)$ during the measurements. To perform gas absorption measurements with the PhCs, the samples were placed in a custom-built gas cell, which allowed recording transmission spectra of PhCs while purging them with a target gas. Fig. 3 shows a schematic of this gas measurement cell. The PhC sample with a length of $1.5 \mathrm{~mm}$ in transmission direction rests in a custom sample holder inside the gas cell. This sample holder blocks light transmission above the microtube arrays and through the substrate [6].

The MIR detector records the transmitted light as a voltage signal. To calculate the transmittance of a $\mathrm{PhC}$ this transmission signal is compared to a reference background signal. This reference signal was recorded by measuring transmission through a $\mathrm{PhC}$ with removed microtubes. Fig. 4 shows the transmission of a hexagonal microtube array with a lattice pitch of $6.5 \mu \mathrm{m}$. The transmission spectrum reveals that strong transmittance is achieved over a wide wavelength range from 5 to $8 \mu \mathrm{m}$ with peak transmission values between $15-30 \%$ at $5.0,6.2$, and $7.0 \mu \mathrm{m}$ respectively.

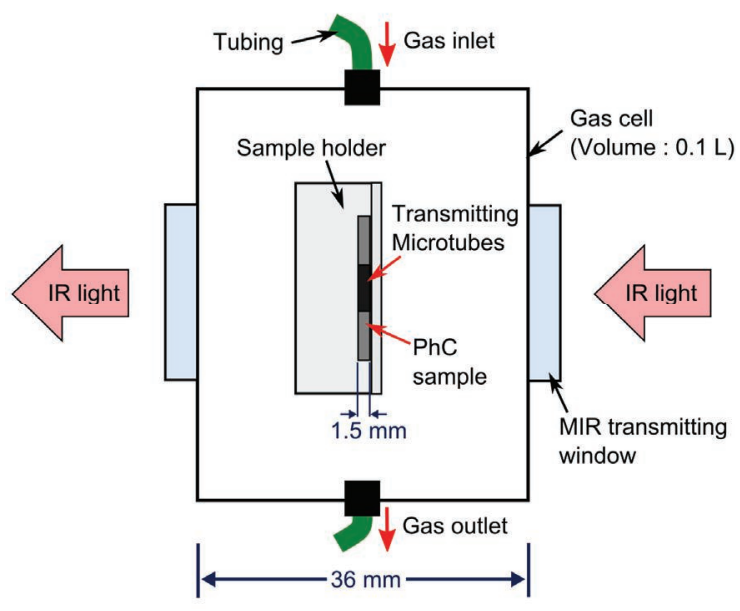

Fig. 3: Schematic of the gas cell for PhC

transmission measurement in the presence of gases. The PhC is built in a sample holder in the center; the black part of the PhC sample contains the microtube arrays.

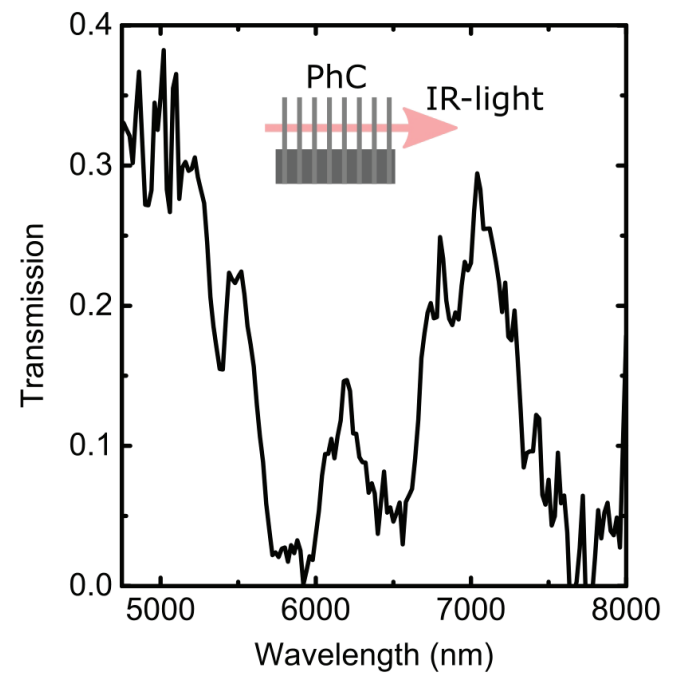

Fig. 4: Transmission spectrum of a hexagonal microtube array with a pitch of $6.5 \mu \mathrm{m}$ in the range from 5-8 $\mu \mathrm{m}$.

\section{Slow light enhanced gas absorption: theory and simulations}

The gas sensor that is presented in this work uses slow light effects in a PhC structure to enhance the absorption of light by gas molecules. Slow light refers to the property of PhCs to transmit light at certain frequencies with very low group velocities $v_{g}$. Light propagation through a $\mathrm{PhC}$ is governed by its photonic band structure. The group velocity of a PhC mode corresponds to the slope of a photonic band at frequency $\omega$ and wavevector $\mathbf{k}$, according to:

$$
v_{g}=\frac{d \omega}{d k}
$$


Low group velocities occur at local extrema of the band structure, where $v_{\mathrm{g}} \rightarrow 0$ [7]. This effect can be used to enhance gas absorption in the $\mathrm{PhC}$ by prolonging the interaction time between light and gas molecules [8]. Light absorption by gas molecules is described by the LambertBeer law [1]:

$$
I=I_{0} \cdot e^{-\alpha \cdot C \cdot l},
$$

where $I$ is the light transmitted through a gas volume, $I_{0}$ is the incident light, $\alpha$ is the absorptivity of the gas, $C$ is the gas concentration, and $/$ is the pathlength of the light through the gas volume. The slow light effect can be interpreted as an effectively enhanced pathlength $\left(l_{\text {int }}\right)$ of the light through the $\mathrm{PhC}$ of length $I_{\text {PhC }}$, according to:

$$
l_{\text {int }}=\frac{c}{v_{g}} \cdot \xi \cdot l_{P h C},
$$

where $c$ is the speed of light in vacuum and $\xi$ is a correction factor that represents the fraction of the energy density of the electric field that is stored in regions of the $\mathrm{PhC}$ normalized by the total energy density in the PhC [8]:

$$
\xi=\frac{\langle E \mid D\rangle_{\text {gas }}}{\langle E \mid D\rangle_{\text {norm }}},
$$

with $0<\xi<1$. Here, $\mathbf{E}$ is the electric field and $\mathbf{D}$ the energy density of $\mathbf{E}$, with $\mathbf{D}=\varepsilon \mathbf{E}$.

For our gas measurements we used an absorption band between 5.3 and $5.6 \mu \mathrm{m}$ of the hydrocarbon gas propene; our IR light source emits its maximum intensity in this wavelength range. As $\mathrm{PhC}$ we used a hexagonal microtube array with a pitch of $6.5 \mu \mathrm{m}$, a height of $80 \mu \mathrm{m}$, and a microtube wall thickness of $\sim 300 \mathrm{~nm}$. First, we investigated the slow light capabilities of this PhC by calculating its TM band structure along the $\Gamma-\mathrm{M}$ transmission direction, using the MPB software package [9].

Fig. 5a shows the bands in the frequency range from 1.0 to $1.4 \omega a / 2 \pi c$. A flat band dispersion of the $7^{\text {th }} \mathrm{TM}$ band, referred to as $\Sigma 7$, occurs in the wavelength range of the propene absorption band, highlighted in the band diagram by a green shadow. Fig. $5 \mathrm{~b}$ shows that the mode profile of this band is mirror symmetric in transmission direction, i.e. light with a plane wave like symmetry can couple into this mode. The corresponding correction factor of the slow light mode is $\xi_{\Sigma 7}=0.59$, suggesting a strong concentration of the E-field in the gas-open parts of the PhC.

a) $\mathrm{PhC}, 6.5 \mu \mathrm{m}$

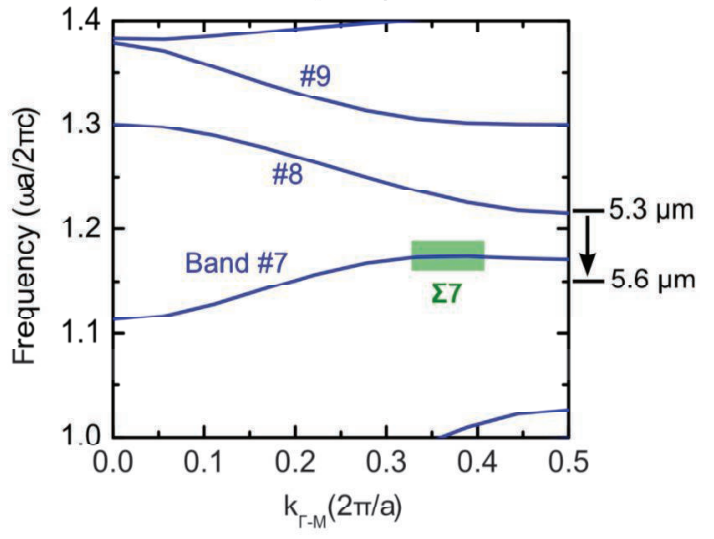

b)

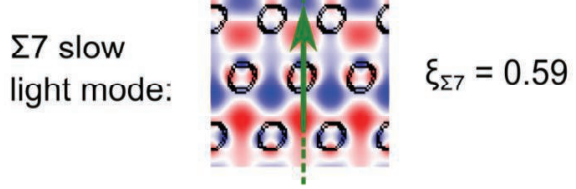

Fig. 5: a) TM bandstructure of a hexagonal PhC along the $\Gamma-M$ direction (pitch $6.5 \mu \mathrm{m}$ ). A flat band dispersion in the wavelength range of the propene absorption band from 5.3 to $5.6 \mu \mathrm{m}$ occurs in the $7^{\text {th }}$ band ( $\Sigma 7)$. b) Color coded magnitude of the E-Field for de $\Sigma 7$ mode and corresponding correction factor $\xi_{\Sigma 7}$. The green arrow corresponds to the propagation direction.

\section{Slow light enhanced gas absorption: experimental results}

The measurement scheme in Fig. 3 was used to investigate the enhancement of the gas absorption due to slow light in the PhC. Gas absorption spectra of propene were determined by dividing the transmission spectra of propene (at $30 \%$ ) and $\mathrm{N}_{2}$ flushed gas cells. $\mathrm{N}_{2}$ shows no absorption features in the target wavelength range. The measurements were performed with a $\mathrm{PhC}$ built into the gas cell and, as a reference, with an empty gas cell without a PhC. To estimate the error of the absorption measurements, every step was repeated three times. Fig. 6 shows the resulting absorption curves. Enhanced absorption due to the PhC sample can be observed at $5400 \mathrm{~nm}$, with an average signal drop from $0.92 \%$ to $0.89 \%$. This wavelength corresponds to the $\Sigma 7$ slow light mode in Fig. $5 b$.

Enhanced absorption due to slow light only occurs inside the $\mathrm{PhC}$ structure, while gas absorption also occurs between the $\mathrm{PhC}$ and the IR transmissive windows on both ends of 
the gas cell. Accounting for the geometry, we use a modified version of the Lambert Beer equation to describe the enhanced absorption:

$$
I=I_{0} \cdot e^{-\alpha C\left(l-l_{P h C}+l_{\text {int }}\right)}
$$

with $l_{\text {int }}$ being the effectively enhanced absorption path length in the PhC (see Equation 4). Combining Eq. 6 with Eq. 3 provides us with a term for $l_{\text {int: }}$ :

$$
l_{\text {int }}=\left[\frac{\ln \left(I_{P h C}\right)}{\ln \left(I_{G C}\right)}-1\right] \cdot l+l_{P h C},
$$

with $I_{\mathrm{PhC}}$ and $\mathrm{I}_{\mathrm{GC}}$ being the absorption ratios $\mathrm{I} / \mathrm{I}_{0}$ for a gas cell with PhC (red curve in Fig. 6) and a reference empty cell (black curve in Fig. 6).

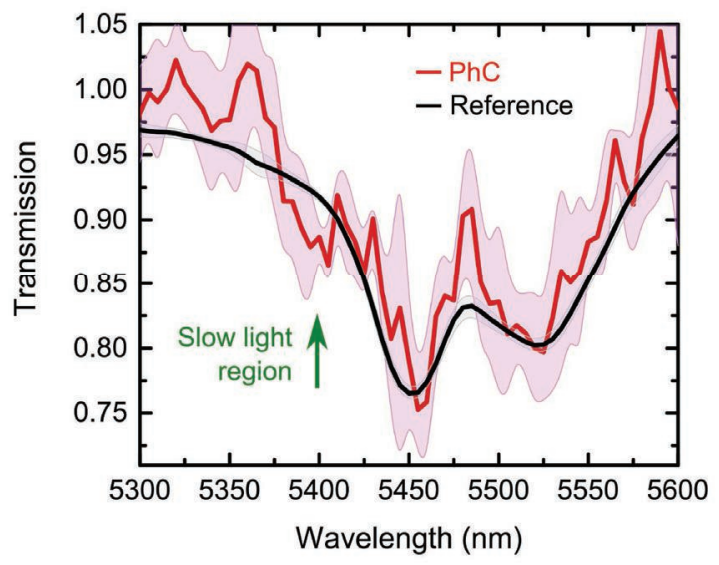

Fig. 6: Comparison of absorption spectrum with a PhC built in the gas cell to a reference measurement with an empty cell. The PhC measurement shows a drop of the absorption signal at $5400 \mathrm{~nm}$ (green arrow).

The enhancement of the gas absorption inside the $\mathrm{PhC}$ corresponds to $l_{\text {int }} / l_{P h C}$ with a corresponding slow group velocity of:

$$
v_{g}=\xi \cdot \frac{l_{P h C}}{l_{\mathrm{int}}} \cdot c
$$

Equation 7 and 8 were used to calculate the enhanced absorption and $v_{\mathrm{g}}$ for the reduced transmission in Fig. 6. At $5400 \mathrm{~nm}$ we calculate an enhancement of the gas absorption of $l_{P h C} / l_{\text {int }}=10.5 \pm 2.1$ and a slow light group velocity of $v_{g}=(0.06 \pm 0.01) c$ in the PhC. This means that the PhC structure of only $1.5 \mathrm{~mm}$ in length substitutes an interaction length of $\sim 15 \mathrm{~mm}$ in a conventional gas spectrometer, thus allowing for a size reduction of one order of magnitude.

\section{Conclusion}

We have presented a method to produce photonic microstructures and demonstrated, that they are able to enhance the absorption of light in a spectroscopic gas sensor. These microstructures consist of an array of high aspect ratio microtubes that show high transmittance in the MIR range. The effect of slow light is used to prolong the interaction time in the $\mathrm{PhC}$, leading to an effectively enhanced pathlength of the light through the gas volume. Enhanced gas absorption by roughly a factor of 10 was observed due to a reduced group velocity in the $\mathrm{PhC}$ of $\sim 0.6 \mathrm{c}$.

We gratefully acknowledge financial support by the EU under grant FP7-257488 (project name: e-BRAINS).

\section{References}

[1] J. Hodgkinson, R. P. Tatam, Optical gas sensing: a review, Measurement Science and Technology 24, 012004 (2013); doi: 10.1088/09570233/24/1/012004

[2] D. Pergande, T. M. Geppert, A. v. Rhein, S. L. Schweizer, R. B. Wehrspohn, S. Moretton, A. Lambrecht, Miniature infrared gas sensors using photonic crystals, Journal of Applied Physics 109, 083117-083117 (2011); doi: 10.1063/1.3575176

[3] C. Kraeh, A. Popescu, M. Schieber, H. Hedler, T. Bieniek, G. Wielgoszewski, M. Moczała, J. Finley, Fabrication of high aspect ratio microtube arrays for $2 \mathrm{~d}$ photonic crystals, Materials Research Express 1, 026201 (2014); doi: 10.1088/2053$1591 / 1 / 2 / 026201$

[4] V. Lehmann, Electrochemistry of Silicon: Instrumentation, Science, Materials and Applications, Wiley-VCH (2002)

[5] V. Lehmann, U. Grüning, The limits of macropore array fabrication, Thin Solid Films 297, 13-17 (1997); doi: 10.1016/S0040-6090(96)09478-3

[6] C. Kraeh, J. L. Martinez-Hurtado, M. Zeitlmair, A. Popescu, H. Hedler, J. J. Finley, Strong transmittance above the light line mid-infrared 2D photonic crystals, submitted (2015)

[7] M. Ghebrebrhan, M. Ibanescu, S. G. Johnson, M. Soljaćić, J. D. Joannopoulos, Distinguishing zerogroup-velocity modes in photonic crystals, Physical Review A 76, 063810 (2007); doi: 10.1103/PhysRevA.76.063810

[8] N. A. Mortensen, S. Xiao, Slow-light enhancement of beer-lambert-bouguer absorption, Applied Physics Letters 90, 141108 (2007); doi: 10.1063/1.2720270

[9] S. G. Johnson, J. D. Joannopoulos, Blockiterative frequency-domain methods for maxwell's equations in a planewave basis, Optics Express 8, 173-190 (2001); doi: 10.1364/OE.8.000173 